\title{
Tailoring molecular self-organization by chemical synthesis: Hexaphenylbenzene, hexa-peri-hexabenzocoronene, and derivatives on $\mathrm{Cu}$ (111)
}

\author{
Leo Gross and Francesca Moresco* \\ Institut fuir Experimentalphysik, Freie Universitatt Berlin, Arnimallee 14, D-14195 Berlin, Germany
}

Pascal Ruffieux

Swiss Federal Laboratories for Materials Testing and Research, Überlandstrasse 129, 8600 Dübendorf, Switzerland

André Gourdon and Christian Joachim

The Nanoscience Group, CEMES-CNRS, 29 Rue J. Marvig, P.O. Box 94347, F-31055 Toulouse Cedex, France

Karl-Heinz Rieder

Institut für Experimentalphysik, Freie Universität Berlin, Arnimallee 14, D-14195 Berlin, Germany

(Received 5 October 2004; published 25 April 2005)

\begin{abstract}
A low-temperature scanning tunneling microscopy (LT-STM) study of four slightly different hydrocarbons based on hexaphenylbenzene (HPB) and hexa-peri-hexabenzocoronene (HBC) on the $\mathrm{Cu}(111)$ surface at submonolayer coverage is presented. All molecules show a commensurate monolayer structure, with significant differences in structure and growth mode. We find that the long range order and the size of defect free domains increase when the intermolecular interaction becomes stronger with respect to the molecule-substrate interaction. Moreover, we can assign adsorption and self-ordering properties to the different chemical groups within the molecules.
\end{abstract}

DOI: 10.1103/PhysRevB.71.165428

PACS number(s): 68.37.Ef, 81.07.Nb

\section{INTRODUCTION}

Well ordered interfaces are of great importance for the application of organic molecules in devices such as lightemitting diodes and field-effect transistors. Self-ordering of organic molecules plays a key role on the way towards the fabrication of ordered molecular thin films and nanostructures. ${ }^{1}$ It is therefore of fundamental interest to gather knowledge about the structure and bonding of molecules on metal and semiconductor surfaces. The thin film growth and the formation of ordered monolayers of organic molecules on metal and insulator surfaces have been intensely studied over the last decade. ${ }^{1-9}$ It has been found that the self-ordering process sensitively relies on the interplay between molecule-substrate and intermolecular forces. For supramolecular self-assembly, a domination of the noncovalent molecule-molecule interaction over molecule-substrate interactions is assumed, while the substrate provides certain adsorption positions like a checkerboard. ${ }^{3,9,10}$ However, further detailed knowledge on the correlation between molecular geometry and molecular epitaxial growth is desirable for the directed synthesis of molecules for applications in organic film technology.

Hexa-peri-hexabenzocoronene (HBC) and its derivatives have been shown to be of great technological interest for the application in light-emitting devices, organic film photovoltaic technology, ${ }^{11}$ and molecular electronics. ${ }^{12-14}$ Selforganization of hexaalkyl-substituted derivates of HBC into a columnar mesophase in organic solvents has been demonstrated, leading to one-dimensional conductors with a very high charge carrier mobility, i.e. molecular nanowires. ${ }^{13}$ Columnar stacking of HBC molecules on metal surfaces has also been reported for organic molecular beam epitaxy (OMBE) in UHV. ${ }^{4}$ Recently, discrete tubular self-assembled nanotubes have been grown from HBC derivatives. ${ }^{14}$

We chose the symmetry of the investigated systems to meet the requirements for epitaxial growth with single domain orientation on the $\mathrm{Cu}(111)$ surface. Like the substrate, the investigated molecules are all showing a sixfold symmetry. The molecules are hexaphenylbenzene ( $\mathrm{HPB}, \mathrm{C}_{42} \mathrm{H}_{30}$ ), hexa-peri-hexabenzocoronene $\left(\mathrm{HBC}, \mathrm{C}_{42} \mathrm{H}_{18}\right)$, hexa-tertbutyl-hexabenzocoronene (HB-HBC, $\mathrm{C}_{66} \mathrm{H}_{66}$ ), and hexa-tertbutyl-hexaphenylbenzene (HB-HPB, $\mathrm{C}_{66} \mathrm{H}_{78}$ ). The corresponding structure models are shown in Fig. 1.

In the following we describe the self-ordering and submonolayer growth of the four molecules. By comparison we can address structural properties and molecular behavior upon adsorption to certain chemical properties of the molecules. We explain how the variations in the molecular geometry affect lateral ordering, island growth, and molecular adsorption geometry.

\section{EXPERIMENT}

Experiments were carried out in an ultrahigh-vacuum chamber with a base pressure of $10^{-10} \mathrm{mbar}$, using a homebuilt low temperature STM, described elsewhere. ${ }^{15}$ The sample was cleaned by several cycles of neon-ion sputtering $(1.3 \mathrm{keV}, 20 \mathrm{~min})$ and subsequent annealing to $770 \mathrm{~K}$ for 5 min.

The molecules were sublimed from Knudsen cells (Kentax TCE-BS) and the dosage was checked via a quartzcrystal-microbalance. The molecules were dosed at a rate of about $10^{-4}$ monolayer/s at an ambient pressure of 


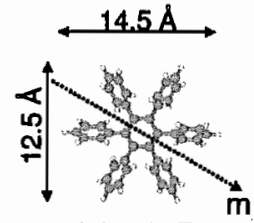

(a) HPB

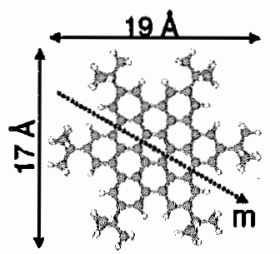

(c) HB-HBC

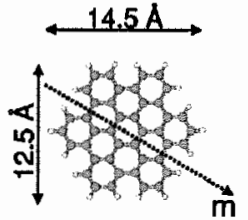

(b) $\mathrm{HBC}$

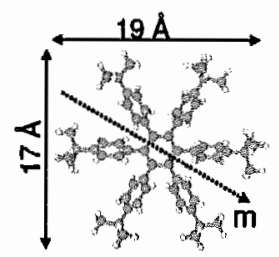

(d) HB-HPB
FIG. 1. Structure models and van der Waals dimensions of the investigated molecules. The dashed arrows indicate the molecular axis $\mathbf{m}$. (a) HPB, due to steric interaction the outer phenyl rings are tilted around the sigma bonds to the central benzene ring; (b) HBC, an entirely planar aromatic system; (c) HB-HBC; and (d) HB-HPB are both equipped with six additional tert-butyl groups at the outer aromatic rings and have the same internal structure as (b) and (a), respectively.

$10^{-9}$ mbar. Evaporation times were 5-15 min, leading to coverages of $1 \%-10 \%$ of a full monolayer (ML) as checked by STM. We define $1 \mathrm{ML}$ as the amount of molecules, that would cover the sample completely, assuming the densest observed monolayer structure. The evaporation temperatures for the different molecules were $420 \mathrm{~K}, 620 \mathrm{~K}, 570 \mathrm{~K}$, and $520 \mathrm{~K}$ in the case of HPB, HBC, HB-HBC, and HB-HPB, respectively. The $\mathrm{Cu}(111)$ substrate was maintained at $(320 \pm 10) \mathrm{K}$ during sublimation to allow molecular diffusion and island formation. This temperature was held for about 10 min, subsequently the sample was cooled using liquid $\mathrm{He}$ and transferred to the STM without breaking the vacuum. Measurements were performed at a temperature of $7 \mathrm{~K}$. The bias voltage always refers to the sample with respect to the tip and the orientation of the sample is identical for all measurements. All shown images have been recorded in constant current mode.

\section{EXPERIMENTAL RESULTS}

\section{A. Hexaphenylbenzene}

The hexaphenylbenzene molecule [HPB, $\mathrm{C}_{42} \mathrm{H}_{30}$, Fig. 1(a)] consists of six phenyl groups, each one connected to the central benzene ring by a $\sigma$-bond. Steric hindrance between the outer phenyl groups forbids a planar geometry for the molecule, in contrast to the similar, but planar HBC. The phenyl groups of HPB are rotated around the $\sigma$-bonds, resulting in a propellerlike shape of the molecule. An effect of the nonplanarity can already be seen in the sublimation temperature of HPB, which is about $200 \mathrm{~K}$ lower than for the planar HBC, since only in the latter case strong van der Waals bonding between parallel layers of molecules, similar to the case of graphite, is possible.

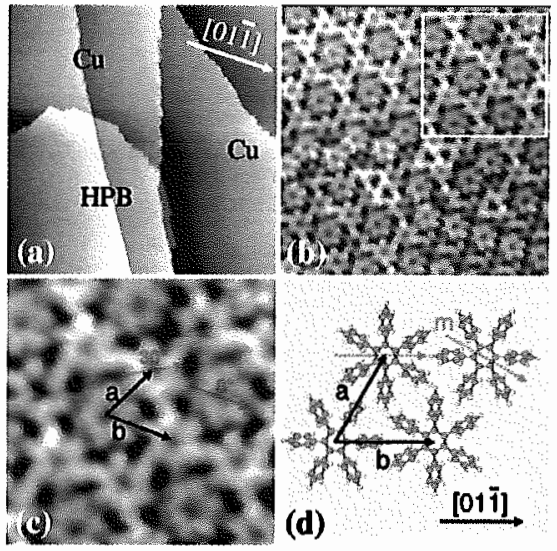

FIG. 2. (Color online) STM measurements of HPB on $\mathrm{Cu}(111)$. (a) Typical overview $\left(V=-1 \mathrm{~V}, I=0.2 \mathrm{nA}, 800^{2} \AA^{2}\right)$, the molecules form large islands sometimes covering several $\mathrm{Cu}$ terraces. (b) Monolayer structure $\left(V=-0.8 \mathrm{~V}, I=0.5 \mathrm{nA}, 100^{2} \AA^{2}\right)$. Molecules appear with different contrast corresponding to the molecular orientation. In the cut-out (c) the molecular orientation, i.e., the angle between [01 $1 \overline{1}$ ] direction and $\mathbf{m}$ (dashed arrows), is indicated for some molecules. (d) Structure model.

When a few percent of a ML of HPB is evaporated on $\mathrm{Cu}(111)$, extremely large molecular islands form. A typical overview showing a part of a HPB island is shown in Fig. 2(a). The islands extend often over several terraces and covered terrace steps are straightened due to the presence of the molecules. Such terrace steps and the borders of molecular islands run preferably along the close-packed directions of the substrate. In general, the adsorption site at step edges is not preferred to molecular agglomerations and molecular islands are found although the step edges are not saturated.

STM images of the monolayer structure with submolecular resolution, [Figs. 2(b) and 2(c)], are obtained sometimes due to a modified tip (in this case molecules or part of them have been picked up to the tip). Single molecules appear as six maxima, corresponding to the six outer tilted phenyl groups around a central minimum at the position of the central planar benzene ring. The distance of two opposite maxima within a molecule is $(10 \pm 1) \AA$ in accordance with the distance of the phenyl rings inside a molecule. The apparent average height of the molecular layer is $2 \AA$. The molecules form a hexagonal structure. The unit cell vectors determined by STM are $|\mathbf{a}|=|\mathbf{b}|=(12.8 \pm 0.3) \AA$, the angle between $\mathbf{a}$ and $\mathbf{b}$ is $\Phi=(60 \pm 2)^{\circ}$ and the angle between $\mathbf{a}$ and the [01 $\overline{1}]$ direction is $\Theta=(0 \pm 2)^{\circ}$. Therefore we propose a $(5 \times 5) R 0^{\circ}$ structure. However, there exists a larger unit cell consisting of several molecules, since molecules show two different kinds of contrast in STM images. This contrast change has its origin in the orientation of the molecular axis m which may be either rotated $\sim 0^{\circ}$ or $\sim 30^{\circ}$ with respect to

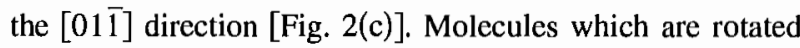
about $30^{\circ}$ usually show a larger apparent height than the molecules with $0^{\circ}$ rotation, although the submolecular contrast does strongly depend on tip conditions and tunneling voltage. The orientation of some molecules is indicated in 


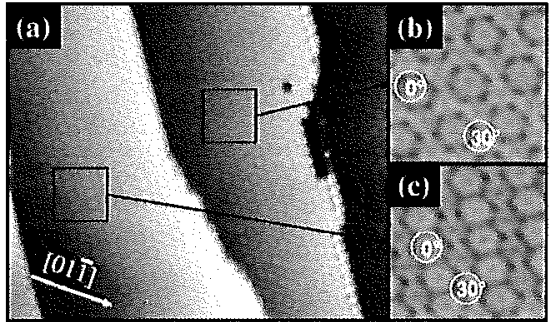

FIG. 3. STM measurements of HPB $(V=1 \mathrm{~V}, I=0.2 \mathrm{nA})$. (a) Overview showing several HPB covered terraces. Molecules that are rotated $0^{\circ}$ and $30^{\circ}$ appear with different contrast forming molecular superstructures, mainly a $(\sqrt{3} \times \sqrt{3})$ superstructure, as in (b) and rarely a $(2 \times 1)$ superstructure appears as shown in (c). In (b) and (c) the molecular orientation $\mathbf{m}$ of different molecules is indicated.

Fig. 2(c). In the preferred supermolecular structure, the molecules with $30^{\circ}$ rotation form a honeycomb structure with the $0^{\circ}$ rotated molecules inside, thus resulting in a $(\sqrt{3} \times \sqrt{3})$ super unit cell as can be seen in Fig. 2(c). A structure model based on the experimental results is shown in Fig. 2(d).

Different molecular superstructures can be observed. A larger covered region is shown in Fig. 3(a) and it can be seen that the $(\sqrt{3} \times \sqrt{3})$ structure [shown in Fig. 3(b)] is dominating in most parts. However, long range order of the superstructure is poor and many defects and antiphase domain boundaries can be observed. Some regions of Fig. 3(a) show a different superstructure with a $(1 \times 2)$ unit cell as shown in Fig. 3(c).

\section{B. Hexa-peri-hexabenzocoronene}

Hexa-peri-hexabenzocoronene [HBC, $\mathrm{C}_{42} \mathrm{H}_{18}$, Fig. 1(b)] is a molecule well characterized in literature. Monolayer structures on $\mathrm{Cu}(111)$ (Ref. 4) and $\mathrm{Au}(111)$ (Refs. 4,16) have been described in detail. HBC is a full planar aromatic system, known to grow commensurate on $\mathrm{Cu}(111)$ in case of a saturated monolayer coverage. ${ }^{4}$ The distance of two neighboring carbon rings inside $\mathrm{HBC}$ is $2.5 \AA$, fitting very accurately the nearest-neighbor distance of $\mathrm{Cu}(111)(2.55 \AA)$.

A typical STM image of a sample covered with a few percent of a monolayer of HBC is shown in Fig. 4(a). The step edges are covered with molecules and isolated molecules can be seen on the terraces. Preparations with lower coverage lead to partially covered step edges and uncovered terraces, indicating that the molecules are mobile on the surface at $T=320 \mathrm{~K}$ and preferentially adsorb at step edges. Adsorption on the terraces takes place only if the coverage is high enough to saturate all step edges [as in Fig. 4(a)]. In this case the molecules on the terraces are always found separated. Even annealing to $470 \mathrm{~K}$ after evaporation, in order to increase the molecular mobility, does not lead to any molecular agglomeration. We find that the recently reported structure ${ }^{4}$ forms only when the first monolayer is completely saturated. The molecules do not form molecular islands in submonolayer coverage although they are mobile on the surface, indicating a lacking of attractive intermolecular forces. The same effect has been observed also for other planar hy-

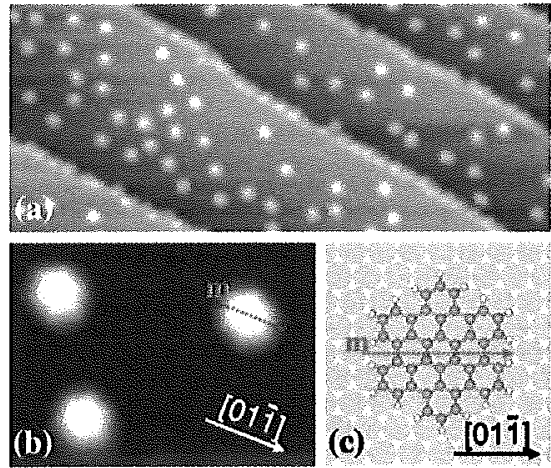

FIG. 4. (Color online) (a) STM image of $\mathrm{HBC}$ on $\mathrm{Cu}(111)$, the step edges are saturated, molecules on the terraces are found isolated. (b) Single molecules $\left(V=-0.1 V, I=0.2 \mathrm{nA}, 90 \times 50 \AA^{2}\right)$. (c) Corresponding adsorption model. The molecular axis $\mathbf{m}$ is indicated.

drocarbons, like for example perylene, ${ }^{5,9}$ coronene $^{5,17}$ and the almost planar decacyclene ${ }^{7}$ on various noble metal surfaces.

Single HBC molecules on a $\mathrm{Cu}(111)$ terrace appear in STM images [Fig. 4(b)] as hexagons with an apparent height of $1.8 \AA$ (at tunneling bias between $-0.5 \mathrm{~V}$ and $0.5 \mathrm{~V}$ ). The lateral dimensions measured from half maximum to half maximum are $14 \AA$ from corner to opposite corner and $13 \AA$ from side to opposite side, respectively, in good correspondence to the van der Waals dimensions of the molecule [Fig. 1(b)]. The orientation of the hexagons is identical for all molecules and reflects the molecular orientation on the surface. The molecules are oriented with the molecular axis $\mathbf{m}$ parallel to the close-packed directions of the $\mathrm{Cu}$ surface, as shown in the model in Fig. 4(c), thus allowing equal positions of all carbon rings inside the HBC molecule with respect to the $\mathrm{Cu}(111)$ substrate. This molecular orientation is the same as found for the full monolayer. ${ }^{4}$

\section{Hexa-tert-butyl-hexabenzocoronene}

The larger hexa-tert-butyl-hexabenzocoronene molecule, [HB-HBC, $\mathrm{C}_{66} \mathrm{H}_{66}$, Fig. 1(c)] is formed by substitution of the six free para position of the HBC core by six tert-butyl $\left(\mathrm{C}_{4} \mathrm{H}_{9}\right)$ groups. The influence of such spacer groups has been already studied on the decacyclene (DC) molecule, which has been investigated with and without spacer legs on $\mathrm{Cu}(110) .^{6,7}$ The spacer groups are known to elevate the molecular core with respect to the planar metal surface, decreasing the surface interaction of the molecular core and therefore lowering the diffusion barrier. On the other hand, adsorption geometry with a large overlap between molecular $\pi$-orbitals and metal surface is preferred. The overlap can be increased, despite the spacer legs, by adsorption at step edges or by formation of nanostructures under the molecular core, as observed for HB-DC on $\mathrm{Cu}(110)$ (Ref. 6) and for Lander molecules on $\mathrm{Cu}(110)$ (Ref. 18) and $\mathrm{Cu}(211){ }^{18}$

Figure 5(a) shows a $\mathrm{Cu}(111)$ sample with a coverage of $\sim 0.1 \mathrm{ML}$ of HB-HBC. The step edges are saturated and molecules on the terraces are found separated or in small islands with a typical size of $10-10^{2}$ molecules. The $\mathrm{Cu}$ ter- 


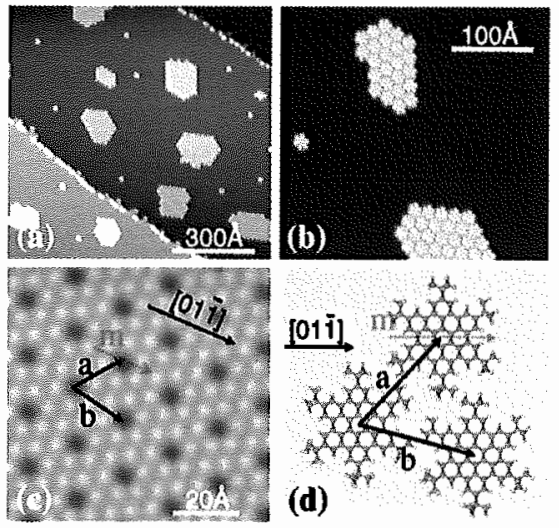

FIG. 5. (Color online) STM measurements of HB-HBC on $\mathrm{Cu}(111)$. (a) Typical overview after preparation at $320 \mathrm{~K}$. Several small islands and isolated molecules can be seen. (b) Two islands of different orientation and one separated molecule. (c) A molecular covered region with indicated unit cell vectors and molecular axis m. (d) Structure model.

races are equally covered with molecular islands. In Figs. 5(b) and 5(c) such islands are reported with submolecular resolution. Each molecule shows six maxima corresponding to the six tert-butyl spacer groups of the molecule. In Fig. 5 (c) the molecular unit cell vectors and the orientation $\mathbf{m}$ of the molecular axis are indicated. The molecular axis $\mathbf{m}$ is

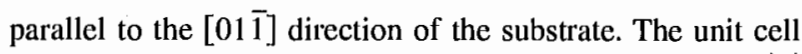
vectors of the molecular layer determined with STM are $|\mathbf{a}|$ $=|\mathbf{b}|=(18.4 \pm 0.3) \AA$. The angle between $\mathbf{a}$ and $\mathbf{b}$ is $\Phi$ $=(60 \pm 2)^{\circ}$ and the angle between $\mathbf{a}$ and the $[01 \overline{1}]$ direction is $\Theta= \pm(14 \pm 3)^{\circ}$. Since no superstructure or modulation is observed and a commensurate structure is possible within the experimental errors we propose a

$$
\left(\begin{array}{cc}
6 & -2 \\
2 & 8
\end{array}\right)
$$

structure, corresponding to values of $|\mathbf{a}|=|\mathbf{b}|=18.4 \AA, \Phi$ $=60^{\circ}$, and $\Theta= \pm 13.9^{\circ}$.

We therefore conclude that the HB-HBC molecules form islands with a $(\sqrt{52} \times \sqrt{52}) R 14^{\circ}$ structure on $\mathrm{Cu}(111)$. The proposed structure model is shown in Fig. 5(c). It follows that islands with two different orientations are possible, namely

$$
\left(\begin{array}{cc}
6 & -2 \\
2 & 8
\end{array}\right) \text { and }\left(\begin{array}{cc}
8 & 2 \\
-2 & 6
\end{array}\right)
$$

In Fig. 5(b) islands of both kind can be seen, in one case the unit cell is rotated $+14^{\circ}$ and in the other case $-14^{\circ}$ with respect to the [011] direction. The different molecular islands can also be recognized in images without molecular resolution [Fig. 5(a)] by observing the preferred direction of their edges, which run in an angle of $+14^{\circ}$ or $-14^{\circ}$ with respect to the close-packed directions of the substrate.
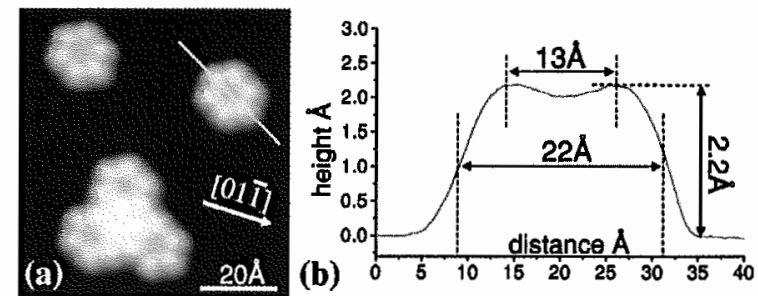

FIG. 6. (a) Two single HB-HBC molecules and a cluster of three molecules. The indicated line displays the position of the line scan shown in (b).

Isolated molecules are shown in Fig. 6(a) and a line scan across a molecule is shown in Fig. 6(b). The distance of two opposing maxima within one molecule is $13 \AA$, corresponding to the distance of the tert-butyl groups inside the molecule. The board of the molecule has an apparent height of $2.0 \AA$.

All molecules are oriented in the same direction with respect to the $\mathrm{Cu}(111)$ substrate, with the molecular axis $\mathbf{m}$ parallel to the [01 $\overline{1}]$ direction. This is observed for both isolated molecules and for those forming islands. Since the orientation of the molecular board is the same as for the adsorption of HBC, we believe that the adsorption site of the two molecules is identical.

\section{Hexa-tert-butyl-hexaphenylbenzene}

The hexa-tert-butyl-hexaphenylbenzene molecule, [HB-HPB, $\mathrm{C}_{66} \mathrm{H}_{78}$, Fig. 1(d)] corresponds to a HPB molecule with six additional tert-butyl spacer groups. In submonolayer coverage HB-HPB forms large ordered islands, covering few terraces completely and leaving most $\mathrm{Cu}$ terraces and step edges uncovered. An overview of this situation is shown in Fig. 7(a). The long range order of these molecular structures is found to be very good and defect free islands of about $1000 \AA$ in diameter are observed. Figures 7 (b) and 7 (c) show the HB-HPB structure with molecular and submolecular resolution, respectively. Like in the case of HPB, the island border lines preferably run along the close-packed directions of the substrate. In contrast to HPB, HB-HPB islands do rarely extend over steps or cover steps. HB-HPB islands are terminated by step edges and usually the islands do not extend to the edge of the step, but a space narrower than one molecular width remains uncovered in front of the step edge (up and down) [see Fig. 7(b)].

Single HB-HPB molecules, as resolved in Fig. 7(c), exhibit six maxima corresponding to the positions of the tertbutyl side groups. Contrary to HB-HBC, the molecular board shows characteristic contrast inside. The central benzene ring is imaged as a distinct minimum.

The monolayer structure of HB-HPB also differs from the structure of HB-HBC, although both molecules are almost identical in size and are equipped with the same side groups. The unit cell vectors of the HB-HPB structure are aligned with the substrate and are measured as $|\mathbf{a}|=|\mathbf{b}|$ $=(18.0 \pm 0.3) \AA$ corresponding to $(7 \times 7) R 0^{\circ}$ growth on $\mathrm{Cu}(111)$. However, the symmetry axis of single molecules inside the monolayer is not aligned with the substrate sym- 

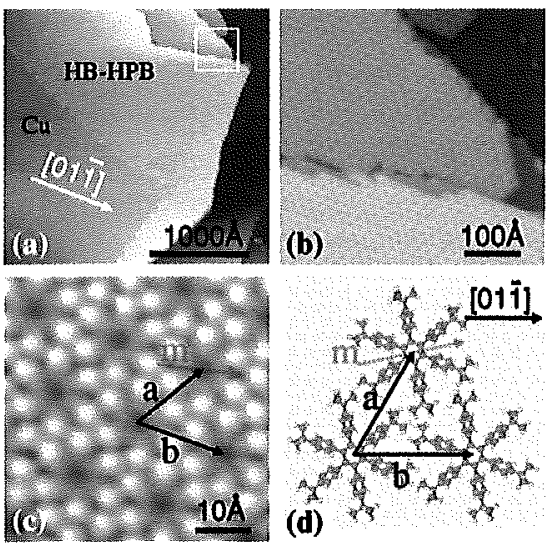

FIG. 7. (Color online) STM measurements of HB-HPB on $\mathrm{Cu}(111)$ for decreasing image sizes $(V=0.8 \mathrm{~V}, I=0.2 \mathrm{nA})$. (a) Molecule covered terraces are identified by Moiré patterns. In (b) single molecules are visible showing typical STM images of the HB-HPB monolayer, where no defects can be seen. (c) HB-HPB monolayer resolved with submolecular resolution. The six tert-butyl groups appear as distinct maxima, the central phenyl ring is observed as a depression. The structure unit cell vectors and molecular axis $\mathbf{m}$ are indicated. (d) Structure model.

metry planes. The molecules are oriented uniformly within one island, with the molecular axis rotated $(11 \pm 3)^{\circ}$ with respect to the $[01 \overline{1}]$ direction of the substrate. Therefore two domains with identical unit cell vectors but different chirality can be observed: One domain in which all molecules are rotated $11^{\circ}$ clockwise and one domain in which all molecules are rotated $11^{\circ}$ anticlockwise with respect to the [01 i] direction. A structure model is shown in Fig. 7(d). Islands with different chirality, i.e., molecular orientation, can be observed in Fig. 8. Due to the propeller shape of the HPB core the HPB and HB-HPB molecules itself show a chirality when adsorbed on the metal surface. However, we cannot distinguish the expected enantiomers. The chirality of the single molecules is probably not connected with the observed chirality of domains, which has its origin in the (clockwise or anticlockwise) rotation of the molecular axis with respect to the $[01 \overline{1}]$ orientation.

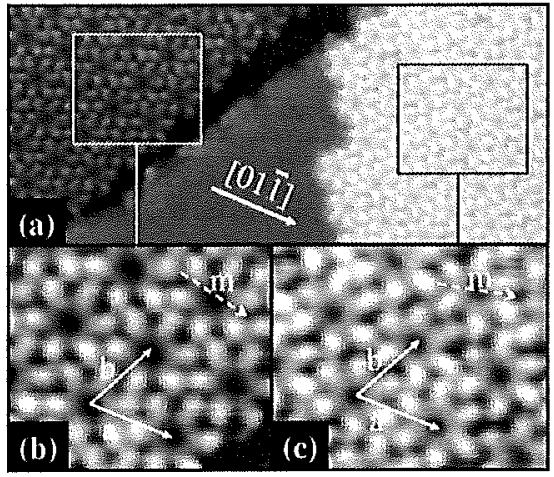

FIG. 8. Two domains of HB-HPB with different chirality. In (a) both domains are shown in one STM image $(V=-0.1 \mathrm{~V}, I$ $=0.2 \mathrm{nA}, 230 \times 110 \AA^{2}$ ). Regions in both domains are shown enlarged in (b) and (c), the unit cells are indicated. Note that the unit vectors of both cells are identical; the domains differ only in the orientation of the molecular axis $\mathbf{m}$ (dashed arrows).

\section{DISCUSSION}

All the investigated molecules form commensurate monolayer structures on $\mathrm{Cu}(111)$. A comparison between the submonolayer growth of all four molecules is shown in Table I. The entirely planar HBC molecule, missing intermolecular van der Waals forces strong enough to form islands in the submonolayer regime, is forced in its hexagonal closepacked structure only for the completely saturated monolayer. The missing of out-of-plane molecular bonds is probably the reason for the small attractive intermolecular interaction, observed for several fully planar hydrocarbons on noble metal surfaces. ${ }^{5,17}$

We find the molecules HBC and HB-HBC always oriented with their molecular axis parallel to the close-packed directions of the $\mathrm{Cu}(111)$ substrate. This can be understood due to the interaction of the planar molecular board, which fits the substrate very accurately in its lattice constant, allowing equal adsorption positions for all carbon rings of the molecular board.

The molecules with HPB core (HPB and HB-HPB), having only one aromatic ring planar to the surface, seem to face only little constraint on molecular orientation due to molecule substrate interactions. HB-HPB forms large molecular

TABLE 1. Comparison of monolayer structures of all four investigated molecules on $\mathrm{Cu}(111)$. The structure of HBC is only formed in the case of a saturated monolayer (Ref. 4).

\begin{tabular}{lcccccc}
\hline \hline Molecule & $\begin{array}{c}\text { Monolayer } \\
\text { structure }\end{array}$ & $\begin{array}{c}\text { Nearest-neighbor } \\
\text { distance }\end{array}$ & $\begin{array}{c}\text { Orientation } \\
\text { of } m(\Phi)\end{array}$ & $\begin{array}{c}\text { Number of } \\
\text { domains }\end{array}$ & $\begin{array}{c}\text { Submonolayer } \\
\text { growth }\end{array}$ & $\begin{array}{c}\text { Preferred } \\
\text { adsorption site }\end{array}$ \\
\hline HBC & $(\sqrt{31} \times \sqrt{31}) R \pm 9^{\circ}$ & $14.2 \AA$ & $0^{\circ}$ & 2 orientational & $\begin{array}{c}\text { separated } \\
\text { molecules }\end{array}$ & step edges \\
HB-HBC & $(\sqrt{52} \times \sqrt{52}) R \pm 14$ & $18.4 \AA$ & $0^{\circ}$ & 2 orientational & $\begin{array}{c}\text { small } \\
\text { islands }\end{array}$ & step edges \\
HBP & $(5 \times 5) R 0^{\circ}$ & $12.6 \AA$ & $0^{\circ} / 30^{\circ}$ & $\begin{array}{c}\text { several } \\
\text { large islands }\end{array}$ & $\begin{array}{c}\text { molecular islands } \\
\text { and step edges } \\
\text { molecular islands }\end{array}$ \\
HB-HPB & $(7 \times 7) R 0^{\circ}$ & $17.9 \AA$ & $11^{\circ}$ & 2 chiral & large islands & molectal \\
\hline \hline
\end{tabular}


domains with uniform molecular orientation. The molecules are tilted by $11^{\circ}$ with respect to the [01 $\left.\overline{1}\right]$ direction, allowing a very dense packing of molecules, which are interlocked. The HB-HPB molecules form a $(7 \times 7)$ structure on $\mathrm{Cu}(111)$, i.e., the unit cell vectors are aligned with the substrate and the packing is denser compared to the HB-HBC structure. For HB-HPB two domains of different chirality are observed (Fig. 8), while there are two domains with different orientation in the case of HBC (Ref. 4) and HB-HBC [Fig. 5(b)].

The HPB molecular axis is rotated either $0^{\circ}$ or $30^{\circ}$ with respect to the [011] direction, leading to $(\sqrt{3} \times \sqrt{3}),(1 \times 2)$ or disordered domains, where the first seems to be the preferred superstructure. The reason for such superstructures is not clear. Obviously, the nearest neighbor distance is wide enough to allow different molecular orientations. Moreover, the switching of the rotation of single molecules in this structure is possible by means of STM manipulation, i.e., when applying voltage pulses of $2.5 \mathrm{~V}$.

In the case of HPB and HB-HPB the molecular orientation originates from the intermolecular interactions. In HB-HPB, the intermolecular interactions are probably $\mathrm{CH}-\pi$ hydrogen bonds between one $\mathrm{CH}$ of a tert-butyl group and the closest phenyl ring of the neighbour molecule. The existence of a fixed adsorption position on the one hand and little constraint of the substrate on the molecular orientation on the other hand, indicate a single pinning center per molecule. Because of the symmetry of the molecule, we suggest that center to be the central benzene ring. In that aspect the situation is similar to the one of PTCDA on $\operatorname{Ag}(111)$, one of the most prominent examples of commensurate organic epitaxy. ${ }^{2,9}$ It has been pointed out that the existence of a single pinning center is one of the reasons leading to the extremely well ordered growth observed for PTCDA. ${ }^{2,9}$ Like PTCDA on $\mathrm{Ag}(111)$ also HB-HPB on $\mathrm{Cu}(111)$ shows almost perfectly ordered commensurate growth.

In the case of HBC and HB-HBC the molecules are always oriented with respect to the substrate, pointing on the existence of several binding centers. The pinning sites of hydrocarbons are most likely located at those aromatic $\pi$ systems which are parallel to the surface. This is supported by the finding that the surface parallel $\pi$ systems are also the main responsible for surface state scattering as shown in the case of Lander molecules $\mathrm{Cu}(111) .^{19}$

The different weighting of molecule-metal with respect to intermolecular forces becomes noticeable in the preferred adsorption sites. In the case of $\mathrm{HBC}$ the domination of adsorbate-substrate interaction leads to no island formation and molecules preferably adsorb at high coordinated sites of the substrate, like step edges and kink sites. In the case of HB-HBC the tert-butyl side groups induce attractive intermolecular van der Waals forces. The preferred adsorption site, however, is still at step edges, but small molecular islands are formed if all step edges are saturated.

Due to the nonplanarity of the HPB board, the moleculemetal interaction is weakened in the cases of HPB and HBHPB. For both molecules the adsorption site is determined by the substrate, causing commensurate growth, but the molecular orientation is mainly determined by the intermolecular interactions. In this aspect, the influence of the intermo- lecular interaction is becoming stronger, as can also be seen in the fact that adsorption at substrate step edges is not preferred to adsorption at molecular islands.

The stronger bonding to the substrate in case of the HBC core compared to HPB could also be measured by means of STM induced lateral manipulation. The minimum resistance needed to manipulate single isolated molecules under equal conditions is 5 orders of magnitude smaller in the case of HBC and HB-HBC compared to HPB and HB-HPB, respectively. Thus the tip has to be about $5 \AA$ closer to the surface for the lateral manipulation of a molecule with HBC core compared to a molecule with HPB core. This is a clear indication of a higher diffusion barrier and a stronger moleculemetal bonding in the case of the HBC core. The fact that manipulation parameters are approximately equal for $\mathrm{HBC}$ compared to HB-HBC on the one hand and HPB compared to HB-HPB on the other hand, indicates little influence of the tert-butyl legs on the molecule-substrate interaction. The para tert-butyl side groups main axis are in the molecules main plane and are not large enough to elevate the molecular board significantly from its equilibrium position with respect to the surface (contrary to the meta di-tert-butyl groups of Lander molecules ${ }^{18,20}$ ).

A clear correspondence between the degree of order and the balancing between molecule-metal and intermolecular interactions can be made out for the investigated molecules: The more the adsorption is governed by intermolecular interaction, the better is the structural order and the larger the size of defect free domains. However, adsorbate-substrate interactions remain important for commensurate growth and the best molecular order is achieved for molecules with a single pinning center.

\section{CONCLUSIONS}

A LT-STM study of the submonolayer growth of four derivates of hexa-peri-hexabenzocoronene on $\mathrm{Cu}(111)$ has been presented, proposing structure models for the observed ordered monolayer structures. The most important results are summarized in Table I. When going down in Table I, the weighting of the force on the molecules gradually shifts from a strong domination of molecule-substrate forces towards the domination of intermolecular interactions. This shift comes along with an improved molecular ordering. The largest defect free domains have been found for HB-HPB, a molecule with one pinning center, allowing the orientation of the molecules to be adjusted by intermolecular forces.

Thanks to the systematic choice of molecules it was possible to assign the properties of adsorbates to specific chemical groups inside the molecule. We find that tert-butyl groups induce intermolecular attraction, while their influence on the molecule-substrate bonding is negligible. The planar aromatic $\mathrm{HBC}$ is strongly bound to the substrate inducing a fixed orientation, while the HPB core binds only at the central position, allowing a rotation of the molecule in the absence of other pinning centers.

\section{ACKNOWLEDGMENTS}

Partial founding by the European Program RTN AMMIST and the Volkswagen Foundation Project "Single Molecule Synthesis" is gratefully acknowledged. 
*Author to whom correspondence should be addressed. Electronic address: francesca.moresco@physik.fu-berlin.de

${ }^{1}$ S. R. Forrest, Chem. Rev. (Washington, D.C.) 97, 1793 (1997); S. I. Stupp, V. LeBonheur, K. Walker, L. S. Li, K. E. Huggins, M. Keser, and A. Amstutz, Science 276, 384 (1997).

${ }^{2}$ K. Glöckler, C. Seidel, A. Soukopp, M. Sokolowski, E. Umbach, M. Böhringer, R. Berndt, and W.-D. Schneider, Surf. Sci. 405, 1 (1998).

${ }^{3}$ O. Marchenko and J. Cousty, Phys. Rev. Lett. 84, 5363 (2000).

${ }^{4}$ P. Ruffieux, O. Gröning, M. Bielmann, C. Simpson, K. Müllen, L. Schlapbach, and P. Gröning, Phys. Rev. B 66, 073409 (2002).

${ }^{5}$ C. Seidel, R. Ellerbrake, L. Gross, and H. Fuchs, Phys. Rev. B 64, 195418 (2001).

${ }^{6}$ M. Schunack, L. Petersen, A. Kühnle, E. Laegsgaard, I. Stensgaard, I. Johannsen, and F. Besenbacher, Phys. Rev. Lett. 86, 456 (2001).

${ }^{7}$ M. Schunack, E. Laegsgaard, I. Stensgaard, and F. Besenbacher, J. Chem. Phys. 117, 8493 (2002).

${ }^{8}$ J. V. Barth, J. Weckesser, N. Lin, A. Dmitriev, and K. Kern, Appl. Phys. A: Mater. Sci. Process. 76, 645 (2003).

${ }^{9}$ M Eremtchenko, J. A. Schäfer, and F. S. Tautz, Nature (London) 425, 602 (2003).

${ }^{10}$ J. V. Barth, J. Weckesser, C. Cai, P. Günter, L. Bürgi, O. Jeand- upeux, and K. Kern, Angew. Chem., Int. Ed. 39, 1230 (2000).

${ }^{11}$ L. Schmidt-Mende, A. Fechtenkötter, K. Müllen, E. Moons, R. H. Friend, and J. D. MacKenzie, Science 293, 1119 (2001).

${ }^{12}$ M. D. Watson, F. Jäckel, N. Severin, J. P. Rabe, and K. Müllen, J. Am. Chem. Soc. 126, 1402 (2004).

${ }^{13}$ A. J. Fleming, J. N. Coleman, A. B. Dalton, A. Fechtenkötter, M. D. Watson, K. Müllen, H. J. Byrne, and W. J. Blau, J. Phys. Chem. B 107, 37 (2003).

${ }^{14}$ J. P. Hill, W. Jin, A. Kosaka, T. Fukushima, H. Ichihara, T. Shimomura, K. Ito, T. Hashizume, N. Ishii, and T. Aida, Science 304, 1481 (2004).

${ }^{15}$ G. Meyer, Rev. Sci. Instrum. 67, 2960 (1996).

${ }^{16}$ M. Troeker, T. Fritz, and H. Proehl, Phys. Rev. B 65, 245422 (2002).

${ }^{17}$ M. Lackinger, S. Griessl, W. M. Heck1, and M. Hietschold, J. Phys. Chem. B 106, 4482 (2002).

${ }^{18}$ L. Gross, F. Moresco, M. Alemani, H. Tang, A. Gourdon, C. Joachim, and K.-H. Rieder, Chem. Phys. Lett. 371, 750 (2003).

${ }^{19}$ L. Gross, F. Moresco, L. Savio, A. Gourdon, C. Joachim, and K.-H. Rieder, Phys. Rev. Lett. 93, 056103 (2004).

${ }^{20}$ F. Rosei, M. Schunack, P. Jiang, A. Gourdon, E. Lagsgaard, I. Stensgaard, C. Joachim, and F. Besenbacher, Science 296, 328 (2002). 